\title{
A Multipoint Liquid Level Sensor Based on Two Twisted Polymer Optical Fibers in a Race-Track Helical Structure
}

\author{
Yingzi Zhang $\mathbb{D}^{1,2}$ Yulong Hou, ${ }^{1,2}$ Yanjun Zhang $\mathbb{D}^{1,2}$ Liang Zhang $\mathbb{D}^{1,2}$ Yanjun ${ }^{1,2} \mathbb{D}^{1,2}$ \\ Xiaolong Gao $\mathbb{D}^{1,2}$ Huixin Zhang, ${ }^{1,2}$ and Wenyi Liu $\mathbb{D}^{1,2}$ \\ ${ }^{1}$ Key Laboratory of Instrumentation Science \& Dynamic Measurement, Ministry of Education, North University of China, \\ Taiyuan 030051, China \\ ${ }^{2}$ Science and Technology on Electronic Test \& Measurement Laboratory, North University of China, Taiyuan 030051, China
}

Correspondence should be addressed to Wenyi Liu; liuwenyi@nuc.edu.cn

Received 27 December 2017; Revised 16 March 2018; Accepted 15 May 2018; Published 4 June 2018

Academic Editor: Banshi D. Gupta

Copyright (c) 2018 Yingzi Zhang et al. This is an open access article distributed under the Creative Commons Attribution License, which permits unrestricted use, distribution, and reproduction in any medium, provided the original work is properly cited.

\begin{abstract}
A multipoint liquid level sensor based on the RI modulation of macrobend polymer optical fiber (POF) couplers is proposed. By twinning two twisted POFs around a race-track column, a series of U-shaped sensing heads are achieved. The output power in the passive fiber declines in a step form while the sensing heads are immersed into liquid in turn. The introduction of UV optical cement in the gap between two POFs improves the side-coupling ratio, increasing the attenuation per step. For the measurement range of $100 \mathrm{~cm}$, the resolution is $2 \mathrm{~cm}$. The sensor can differentiate different liquids with low temperature dependence.
\end{abstract}

\section{Introduction}

Nowadays, the liquid level measurement has attracted great interests because of the high demand in the applications of flood early warning $[1,2]$, fuel supply system $[3,4]$, structural monitoring [5], and so on. In the fields where the liquid level monitoring of several specific points is needed or liquids of different refractive index (RI) are required to be differentiated, many discrete or multipoint liquid level sensors are proposed [6-10]. Compared to their electronic counterparts, optical fiber sensors show good potential in explosive environment due to their nonconductive measurements and electromagnetic immunity [11], broadening the application area.

POFs offer advantages of low cost, easy handling, lightweight, large numerical aperture, and so on in comparison with the glass optical fibers (GOFs) [12, 13]. Most multipoint liquid level sensors are intensity based and achieved by the POFs in different structures. Lin et al. aligned a group of POF segments coaxially, and the coupling power is affected by the refractive index (RI) of medium between two adjacent fiber segments [14]. The measurement range is $250 \mathrm{~cm}$, but the resolution is $25 \mathrm{~cm}$. Mesquita et al. utilized a vertical POF with grooves spaced at every $20 \mathrm{~cm}$ and realized the liquid level measurement of $200 \mathrm{~cm}$ [5]. Jing et al. twined a POF around a race-track column, and the macrobend losses change with the liquid level [15]. The resolution is satisfactory, but the measurement range is just $5 \mathrm{~cm}$. To improve the sensitivity, a POF is not only bended but also laterally polished [16]. However, the resolution is $34 \mathrm{~cm}$ and the fabrication process is complicated compared with the sensors mentioned above.

In our previous work, we proposed a low-cost liquid level sensor based on two twisted POFs twinning around a racetrack column [17]. The side-coupling power is modulated by the RI of the surrounding medium. When the liquid level increases, the side-coupling power decreases fast in the bent section and slow in the straight section, forming a step attenuation. The resolution is determined by the screw pitch and represents the height of a bent and a straight section. When the measurement range is $100 \mathrm{~cm}$, the resolution is $2 \mathrm{~cm}$, and the sensitivities for water are $5.01 \mathrm{nW} / \mathrm{mm}$ in the bent section and $1.99 \mathrm{nW} / \mathrm{mm}$ in the straight section. Thus, when a bent section and a straight section are submerged into 
liquid, the whole variation of output power is $45.84 \mathrm{nW}$. The signal intensity is so weak that increases the detection cost.

In this paper, a cost-effective approach increasing the signal intensity of the multipoint liquid level sensor is proposed. To improve the side-coupling ratio, the UV optical cement is injected into the gap between two POFs that are twisted and twined around a column, of which the cross section is a racetrack form. With the liquid level rising, the output power of the passive fiber declines with a step in the bent section and remains unchanged in the straight section. The measurement range is $100 \mathrm{~cm}$, and the resolution is $2 \mathrm{~cm}$. For a single sensing head consisting of a bent section and a straight section, the whole attenuation of the output power for water is $112.36 \mathrm{nW}$, which is higher than the one of the sensor proposed in [17].

\section{Principle}

As shown in Figure 1, two naked POFs are twisted and bent in the shape of a U, and the UV optical cement is filled into the gap between the two POFs. The diameters of the core and cladding are 980 and $1000 \mu \mathrm{m}$, respectively. For multimode POFs, if the curvature radius is large enough, the ray theory can be applied to replace the modal theory, in which the light will be treated as rays possessing a certain amount of power. When the light ray propagates into the macrobend section of POFs, the total internal reflection is frustrated, and the ray is refracted on the core-cladding interface if the incident angle becomes smaller than the critical angle. The critical angle is defined as $\theta_{\text {cocl }}=\arcsin \left(n_{\mathrm{cl}} / n_{\mathrm{co}}\right)$ where $n_{\text {co }}=1.492$ and $n_{\mathrm{cl}}=1.417$ are the RI of the core and the cladding, respectively. The smaller the curvature radius is, the more rays are refracted. Some refracted rays in the cladding are refracted again on the cladding-environment interface, of which the incident angle is smaller than $\theta_{\text {clen }}=$ $\arcsin \left(n_{\mathrm{en}} / n_{\mathrm{cl}}\right) . n_{\mathrm{en}}$ is the RI of the surrounding medium. It can be deduced that an increase of rays refracted into environment can be achieved by increasing $n_{\mathrm{en}}$ and these refracted lights are called macrobend losses of the fiber. As a result, when the surrounding medium changes from air to liquid, the macrobend losses increase by a large margin and the light power inside the POF decreases accordingly.

For intensity-based sensors, the signal-to-noise ratio (SNR) is an important factor that must be considered. Additional elements are always applied to eliminate the influence of the undesired signal, increasing the complexity of detection. Hence, a macrobend coupler based on POFs is introduced [18]. The active fiber is connected to the light source, of which the macrobend losses are coupled partly to the passive fiber and called the side-coupling power. The sidecoupling ratio is very small because the energy of the light source is mainly concentrated in the core of the active fiber. Thus, by measuring the side-coupling power, the influence of the light source perturbation on the passive fiber can be neglected and the SNR is improved [17]. However, the sidecoupling ratio is so small that a power meter with a high resolution is required. In this paper, we fill the gap between the two POFs with UV optical cement $(n=1.418)$, which means the RI of the surrounding medium between the two POFs

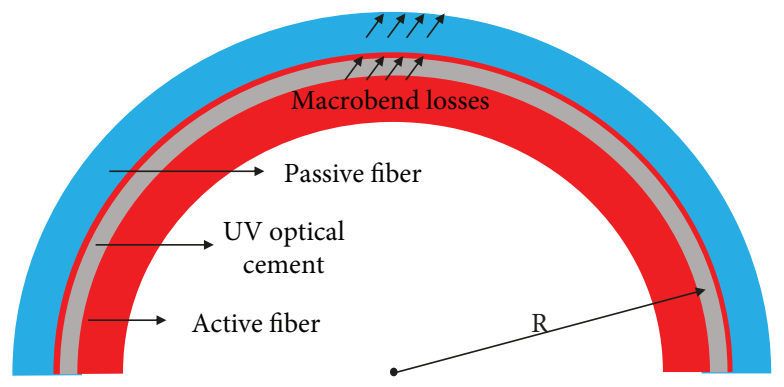

FIgURE 1: A macrobend coupler of two joined POFs.

changes from 1.0 (the RI of air) to 1.418. Therefore, the macrobend losses from the active fiber are increased, and more side-coupling power are coupled into the passive fiber, improving the side-coupling ratio.

If the coupler is immersed into water, the RI increases from 1.0 to 1.333 and more side-coupling power in the passive fiber will be transmitted into the environment medium. The higher the RI of the tested liquid is, the more sidecoupling power are radiated. Therefore, a macrobend POF coupler is sensitive to the RI of the surrounding medium and can distinguish liquids of different RI.

\section{Experimental Setup and Results}

To realize the multipoint liquid level measurement, two twisted POFs (SK40, Mitsubishi) are twined around a race-track column with a screw pitch of $4 \mathrm{~cm}$, forming fifty $\mathrm{U}$-shaped bends acting as sensing head. Thus, the resolution of the sensor which means the distance between two measuring points is $2 \mathrm{~cm}$. The curvature radius of the macrobend section is $1 \mathrm{~cm}$, and the length of the straight section is $2 \mathrm{~cm}$. Then, the UV optical cement (3311, Loctite) is filled into the gap in the macrobend section and cured by the UV curing lamp. Finally, the column is fixed into a container, in which the first sensing head is at the bottom. When the surrounding medium around the sensing head changes from air to liquid, the macrobend losses of the passive fiber increases, reducing the side-coupling power in it. Therefore, with the liquid level ascending, the output power declines step by step related to the number of immersed sensing heads.

The experimental setup of the proposed sensor is depicted in Figure 2. A fiber-coupled LED (M660F1, Thorlabs) with the center wavelength of $660 \mathrm{~nm}$ is utilized as the light source, the output power of which is $30 \mathrm{~mW}$. The optical power meter (PM100USB, Thorlabs) with the resolution of $0.1 \mathrm{nW}$ is adopted to record the output power of the passive fiber under different liquid levels. To improve the signal-to-noise ratio, the insensitive part of the sensor is shielded by the black jacket, and all the experiments are conducted in the dark room.

Figure 3 illustrates output power of the passive fiber when the liquid level changes from 0 to $100 \mathrm{~cm}$. The RI sensing capability of the sensor is evaluated by applying liquids with different $\mathrm{RI}$. Water and $9.1 \% \mathrm{NaCl}$ solution are adopted as the tested liquid. The output signal shows an obvious 


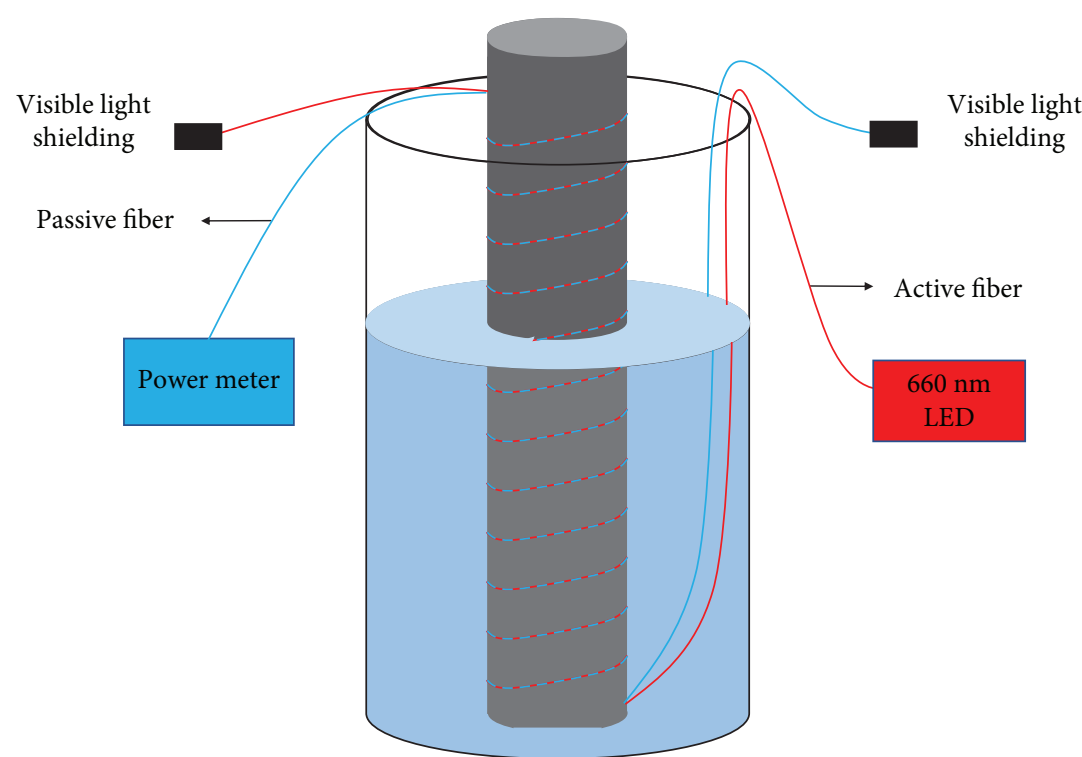

(a)

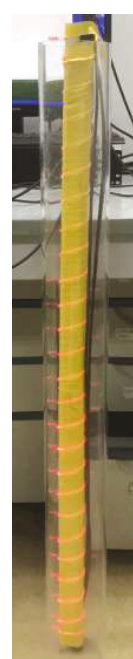

(b)

Figure 2: (a) Setup and (b) photograph of the multipoint liquid level sensor.

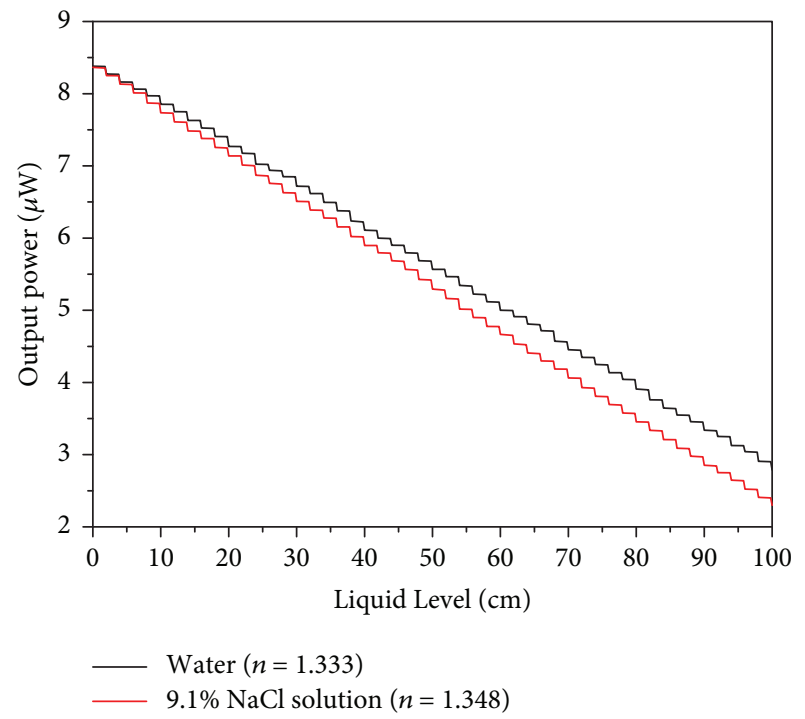

FIGURE 3: Liquid level response to water and $\mathrm{NaCl}$ solution.

decrease when a sensing head is submerged by the liquid. For a sensing head, as the input power of the active fiber is approximately $30 \mathrm{~mW}$, and the output power of the passive one is only a few hundred nanowatt, the attenuation of the active fiber is very small. Since the geometrical structures of these sensing heads are basically the same, the macrobend losses of the active fiber for each sensing head are almost equal. Thus, the decrease in output power for each head is almost the same, forming a step-down output curve with the liquid level rising. For water, the decrease in output power of each sensing head is approximately $112.36 \mathrm{nW}$, which is 2.45 times more than the one of the sensor without the cement in the [17]. From Figure 3, it is obvious that the variation for liquids of different RI is different. When the RI changes from 1.333 to 1.348 , there is an $8.7 \%$ increase in output power per head from 112.36 to $122.18 \mathrm{nW}$, which makes a sensitivity of $0.5 \% /$ RIU. As expected, the tested liquid of higher RI results in a greater change of macrobend losses. At the same time, the output power declines at every point where the sensing head exists no matter what liquid is tested. Therefore, the sensor can distinguish liquids of different RI and the particular liquid levels can be detected independently of the tested liquid.

Temperature dependence of the proposed sensor is investigated, and the result is depicted in Figure 4. In the experiment, the race-track column twined by two twisted POFs was fixed in a container and the liquid level was set to $50 \mathrm{~mm}$. Then, the container was placed on a heater. When the temperature of water rises from 20 to $60^{\circ} \mathrm{C}$, the output power of the passive fiber changes from 5.567487 to $5.741197 \mu \mathrm{W}$, resulting in an overall change of $173.71 \mathrm{nW}$. Compared to the output variation for the change of the liquid level, the influence of temperature is small. Since the temperature also affect the active fiber, we can connect a power meter to the free end of the active fiber to compensate the influence of temperature.

\section{Conclusion}

In summary, two twisted POFs are twined around a racetrack column to achieve the multipoint liquid level measurement. The side-coupling ratio between two POFs is improved by filling UV optical cement into the gap. The macrobend losses are modulated by the RI of the environment medium, and the output power decreases with the liquid level arising. The decrease in a step form occurs every time the sensing head is submerged, and the variation is affected by the RI of the tested liquid. The sensor turns out to be a good 


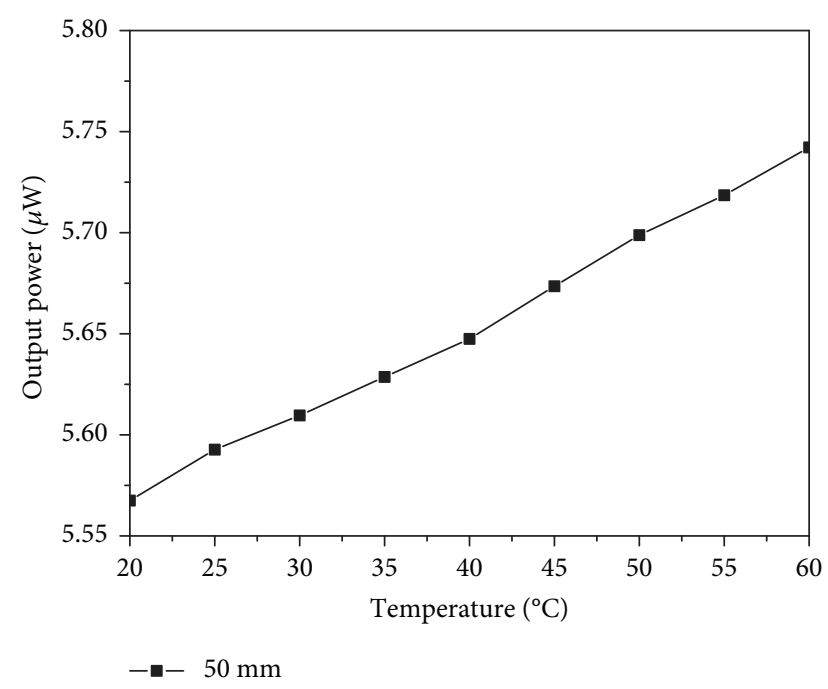

Figure 4: Temperature dependence of the proposed sensor.

design of low cost, simple fabrication process, and low temperature dependence.

\section{Conflicts of Interest}

The authors declare that there is no conflict of interest regarding the publication of this article.

\section{Acknowledgments}

This work was sponsored by the Fund for Shanxi "1331 Project” Key Subject Construction. This work was supported by the National Sciences Foundation of Shanxi Province, China (201701D121065).

\section{References}

[1] K. S. C. Kuang, S. T. Quek, and M. Maalej, "Remote flood monitoring system based on plastic optical fibres and wireless motes," Sensors and Actuators A: Physical, vol. 147, no. 2, pp. 449-455, 2008.

[2] W. A. H. W. M. Asmara and N. H. A. Aziz, "SMS flood alert system," in 2011 IEEE Control and System Graduate Research Colloquium, pp. 18-22, Shah Alam, Malaysia, 2011, IEEE.

[3] G. Onorato, G. Persichetti, I. A. Grimaldi, G. Testa, and R. Bernini, "Optical fiber fuel level sensor for aeronautical applications," Sensors and Actuators A: Physical, vol. 260, pp. 1-9, 2017.

[4] A. Pozo, F. Pérez-Ocón, and O. Rabaza, "A continuous liquidlevel sensor for fuel tanks based on surface plasmon resonance," Sensors, vol. 16, no. 5, p. 724, 2016.

[5] E. Mesquita, T. Paixão, P. Antunes et al., "Groundwater level monitoring using a plastic optical fiber," Sensors and Actuators A: Physical, vol. 240, pp. 138-144, 2016.

[6] V. Svirid and S. N. Khotiaintsev, "Optoelectronic multipoint liquid level sensor for light petrochemical products," in Optoelectronic and Hybrid Optical/Digital Systems for Image and Signal Processing, vol. 4148, pp. 262-269, Lviv, Ukraine, 2000, International Society for Optics and Photonics.
[7] J. E. Antonio-Lopez, J. J. Sanchez-Mondragon, P. LiKamWa, and D. A. May-Arrioja, "Fiber-optic sensor for liquid level measurement," Optics Letters, vol. 36, no. 17, pp. 3425-3427, 2011.

[8] P. Raatikainen, I. Kassamakov, R. Kakanakov, and M. Luukkala, "Fiber-optic liquid-level sensor," Sensors and Actuators A: Physical, vol. 58, no. 2, pp. 93-97, 1997.

[9] I. K. Ilev and R. W. Waynant, "All-fiber-optic sensor for liquid level measurement," Review of Scientific Instruments, vol. 70, no. 5, pp. 2551-2554, 1999.

[10] L. Ren and Q. Yu, "High-accuracy fiber optic level sensor," in Advanced Sensor Systems and Applications, vol. 4920, pp. 362-367, Shanghai, China, 2002, International Society for Optics and Photonics.

[11] P. Antunes, J. Dias, T. Paixão, E. Mesquita, H. Varum, and P. André, "Liquid level gauge based in plastic optical fiber," Measurement, vol. 66, pp. 238-243, 2015.

[12] D. Montero, C. Vázquez, I. Möllers, J. Arrúe, and D. Jäger, “A self-referencing intensity based polymer optical fiber sensor for liquid detection," Sensors, vol. 9, no. 8, pp. 6446-6455, 2009.

[13] H. Golnabi and P. Azimi, "Design and performance of a plastic optical fiber leakage sensor," Optics \& Laser Technology, vol. 39, no. 7, pp. 1346-1350, 2007.

[14] X. Lin, L. Ren, Y. Xu et al., "Low-cost multipoint liquid-level sensor with plastic optical fiber," IEEE Photonics Technology Letters, vol. 26, no. 16, pp. 1613-1616, 2014.

[15] N. Jing, C. Teng, J. Zheng, G. Wang, Y. Chen, and Z. Wang, "A liquid level sensor based on a race-track helical plastic optical fiber," IEEE Photonics Technology Letters, vol. 29, no. 1, pp. 158-160, 2017.

[16] M. Lomer, J. Arrue, C. Jauregui, P. Aiestaran, J. Zubia, and J. M. López-Higuera, "Lateral polishing of bends in plastic optical fibres applied to a multipoint liquid-level measurement sensor," Sensors and Actuators A: Physical, vol. 137, no. 1, pp. 68-73, 2007.

[17] Y. Zhang, Y. Zhang, Y. Hou et al., "An optical fiber liquid level sensor based on side coupling induction technology," Journal of Sensors, vol. 2018, Article ID 2953807, 6 pages, 2018.

[18] Y. L. Hou, W. Y. Liu, S. Su et al., "Polymer optical fiber twisted macro-bend coupling system for liquid level detection," Optics Express, vol. 22, no. 19, pp. 23231-23241, 2014. 


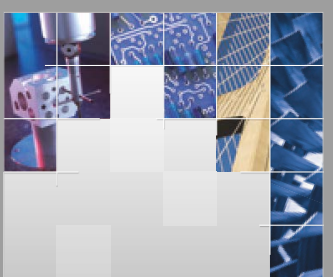

\section{Enfincering}
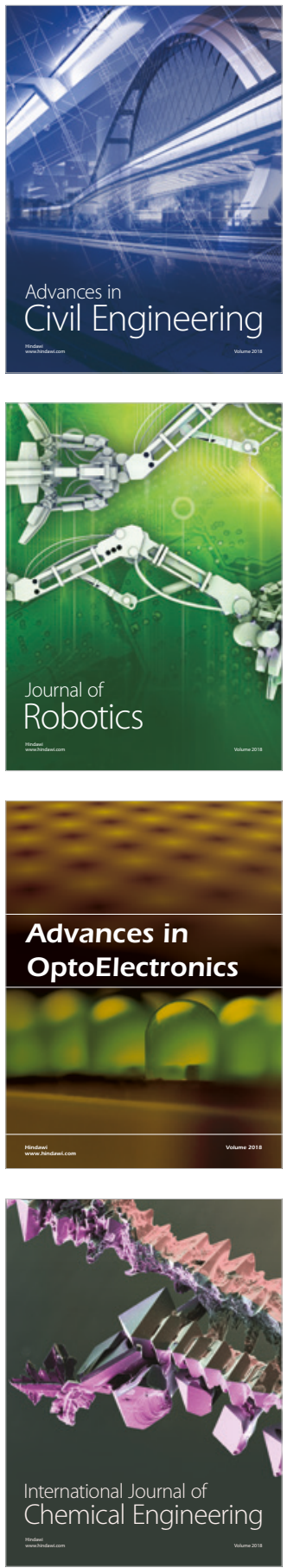

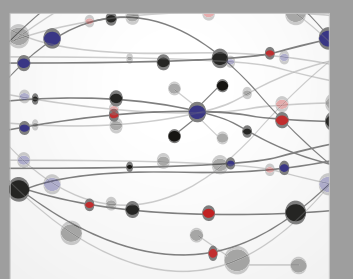

\section{Rotating \\ Machinery}

The Scientific World Journal

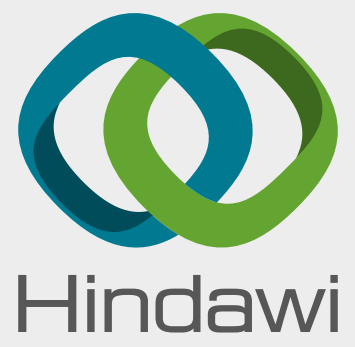

Submit your manuscripts at

www.hindawi.com
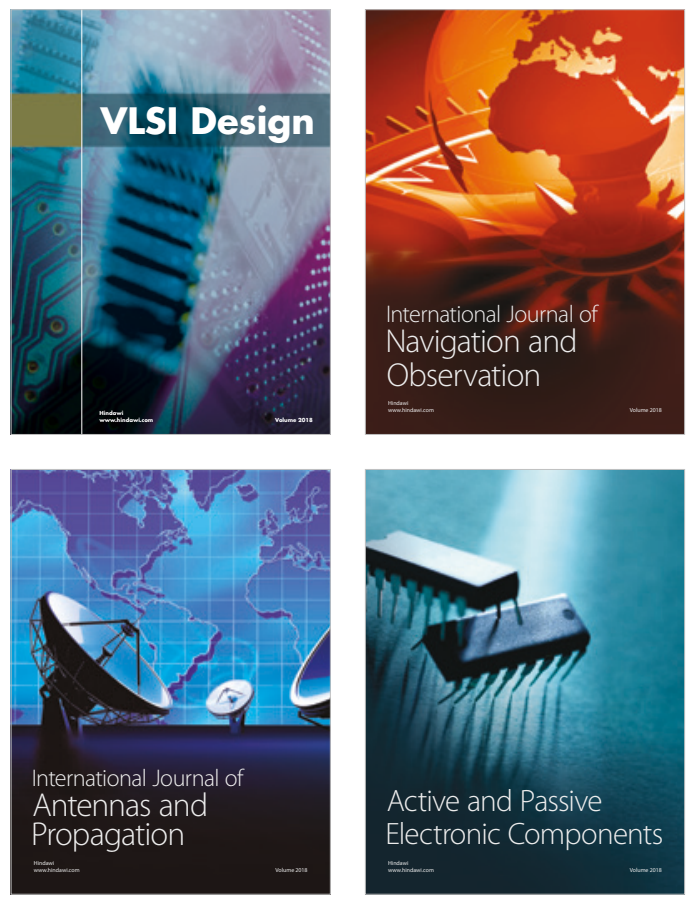
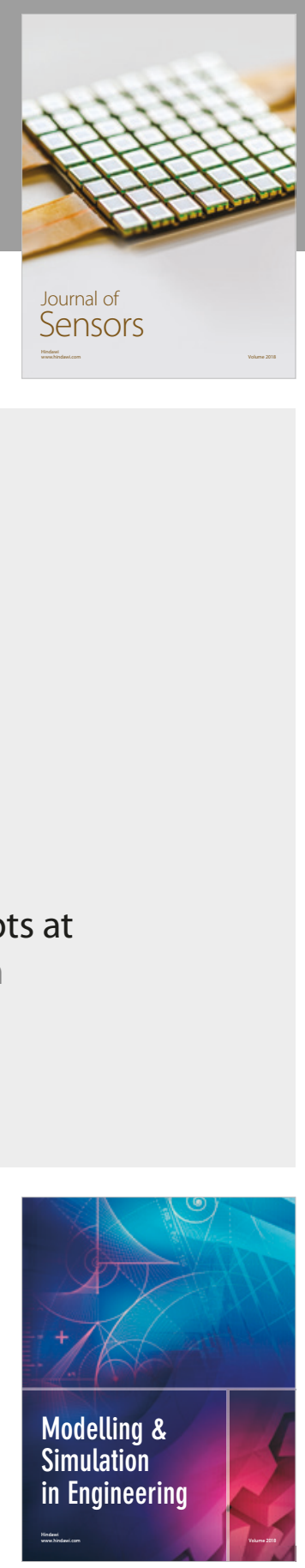

\section{Advances \\ Multimedia}
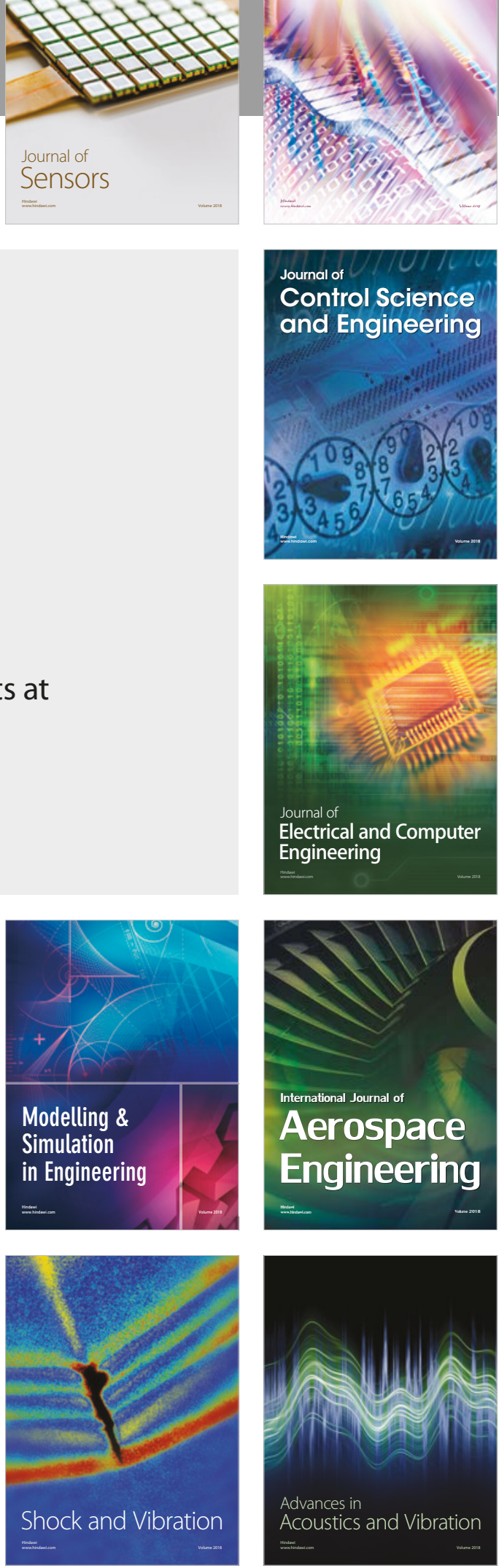